Cahiers $d u$ MONDE RUSSE

\section{Cahiers du monde russe}

Russie - Empire russe - Union soviétique et États indépendants

$59 / 4 \mid 2018$

Varia

\title{
Vicky Davis, Myth making in the Soviet Union and modern Russia
}

Remembering World War II in Brezhnev's hero city

\section{Andreï Kozovoï}

\section{(2) OpenEdition}

Journals

Édition électronique

URL : https://journals.openedition.org/monderusse/10770

DOI : 10.4000/monderusse. 10770

ISSN : $1777-5388$

Éditeur

Éditions de l'EHESS

\section{Édition imprimée}

Date de publication : 1 octobre 2018

Pagination : 665-670

ISBN : 978-2-7132-2747-9

ISSN : $1252-6576$

Référence électronique

Andreï Kozovoï, "Vicky Davis, Myth making in the Soviet Union and modern Russia », Cahiers du monde russe [En ligne], 59/4 | 2018, mis en ligne le 01 octobre 2018, consulté le 08 janvier 2022. URL : http:// journals.openedition.org/monderusse/10770; DOI : https://doi.org/10.4000/monderusse.10770 
Vicky DAVIS

Myth making in the Soviet Union and modern Russia Remembering World War II in Brezhnev's hero city Londres - New York : I.B. Tauris, 2018, 351 p.

Pour Vicky Davis, « si l'on veut vraiment comprendre de quoi une nation ou une communauté sont faites, il est nécessaire de reconnaître l'importance de l'évolution d'un mythe de guerre » (p. 23). C'est lors de sa première visite de la ville de Novorossijsk, port de la mer Noire de la région de Krasnodar en 1999, que l'historienne découvre la cave à vin que le colonel Brežnev, alors responsable de la section politique de la $18^{\mathrm{e}}$ armée du Front Sud, avait transformée en Q.G. militaire — tout un symbole. Au cours de ses recherches, menées en 2010-2016, elle parcourt la ville de fond en comble, passe au crible les archives locales et surtout, collecte de nombreux témoignages : l'histoire orale est l'axe autour duquel se structure son livre, ce qui n'est après tout pas surprenant pour un ouvrage dont le sujet est la mémoire. Son livre est l'aboutissement de ce travail de longue haleine, une étude de cas très fouillée sur le phénomène de « mythe de guerre » en Russie ; une étude de la mémoire à cheval sur les périodes soviétique et postsoviétique.

Novorossijsk avait été occupée par les Allemands partis à la conquête des puits de pétrole du Caucase, qui a représenté par la suite l'épicentre du « culte de la guerre » de Brežnev. Le secrétaire général avait pris part à la libération de la ville en 1943. Détruite à plus de $90 \%$, elle avait finalement obtenu le statut de « ville héros » (gorod-geroj) en 1973. L'épisode de la libération de Novorossijsk fut dépeint dans des Mémoires rédigés pour Brežnev et publiés en 1978 sous le titre de Petite Terre (Malaja Zemlja), référence au petit bout de terre, derrière la plage, sur lequel s'étaient déroulés les combats, entre février et septembre 1943. Le livre et ses épigones ont formé l'ossature du culte de la personnalité du secrétaire général jusqu'à sa mort en 1982, quand le culte et son incitateur furent ridiculisés par les nouveaux hommes au pouvoir. Dans l'ouvrage de Vicky Davis, la libération de Novorossijsk en 1943, puis la visite « historique » de Brežnev dans cette 
ville en 1974, forment deux épisodes fondateurs qui nourrissent le « mythe de la guerre » au niveau local, toujours vivace aujourd'hui ; un mythe qui se maintient non seulement en raison d'une politique de la mémoire actuelle qui s'apparente à un nouveau « culte de la Grande Guerre patriotique », mais aussi grâce au soutien de la population locale. En effet, comme le montre V. Davis dans son livre, « même dans un environnement où la politique mémorielle participe d'une logique verticale, les groupes et les individus peuvent encore impacter les rituels commémoratifs » (p. 5).

Le livre se situe au croisement des études sur Brežnev et la période brejnévienne, qui ont connu un renouveau à partir de 2002, et des travaux sur la mémoire en Russie, notamment liée à la guerre à l'Est, une thématique explorée pour la première fois par Nina Tumarkin (une ancienne doctorante de Richard Pipes) ${ }^{1}$. Inévitablement, l'ouvrage touche aussi à la question plus globale du patriotisme et des mythes politiques dans la Russie actuelle, qui ont également suscité la curiosité de chercheurs, surtout depuis la mue dictatoriale du régime, que l'on date généralement de 2008, année où Putin fit élire son premier ministre, Dimitri Medvedev, au poste de président, pour se faire lui-même nommer à sa place, pour quatre ans ${ }^{2}$. Du point de vue du genre choisi, V. Davis s'inscrit dans une lignée d'auteurs de monographies d'histoire locale, dont le pionnier, on le sait, avait été Merle Fainsod (1907-1972) avec son livre sur Smolensk sous Stalin³

Dans un chapitre introductif consacré à la notion de « mythe de guerre » et les différents ingrédients qui font de Malaja Zemlja un mythe à succès (ville occupée par l'ennemi, débarquement réussi et résistance héroïque pendant plusieurs mois $)^{4}$, la première partie de l'ouvrage est consacrée à la construction du « mythe » dans la littérature. V. Davis met en évidence quatre étapes dans la constitution du mythe : la naissance d'abord, avec l'attribution d'un nom et d'un récit fondateur entamé par les correspondants de guerre dans la presse, à partir de mai 1943 ; puis un travail de rassemblement, entrepris ici par Georgij Sokolov, un officier qui a pris part à la libération de Novorossijsk et qui publie trois recueils de Mémoires (1949, 1954 et surtout 1967, où une soixantaine d'anciens combattants témoignent); l'émergence du mythe « officiel » sous Brežnev, avec la publication de Malaja Zemlja; la convergence complète et l'homogénéisation du mythe en accord avec Brežnev, jusqu'à l'excès, puisqu'un épisode mineur de la Grande Guerre patriotique est hissé au statut de symbole de la guerre tout court, au même titre que la bataille de Stalingrad.

Dans la deuxième partie, V. Davis se penche sur le cas de Brežnev, pour confronter ses deux images de « légende locale » et d'homme d'État d'envergure internationale et expliquer l'érection d'une statue de Brežnev à Novorossijsk, un cas unique dans la Russie postsoviétique, où son nom continue de susciter les moqueries, et se trouve souvent associé à l'idée de stagnation, quand bien même les sondages, depuis de nombreuses années déjà, révèlent que la période brejnévienne, elle, constitue une source apparemment intarissable de nostalgie. Pour comprendre ce paradoxe, V. Davis reconstitue avec moult détails la visite « historique » de Brežnev à Novorossijsk le 7 septembre 1974, et la manière dont cette visite a fini par pratiquement « racheter » Brežnev aux yeux des habitants de la ville qui en ont été témoins et éclipser la mémoire de sa participation à sa libération. 
La raison de la popularité de Brežnev au niveau local tient aussi à une aide financière qui a permis notamment de résoudre le problème d'approvisionnement en eau potable, ainsi que l'appui personnel de Brežnev pour l'installation d'une usine de Pepsi-Cola, boisson gazeuse découverte par les Soviétiques lors de l'Exposition américaine de Moscou de 1959. La statue érigée en l'honneur de Brežnev en 2004 le représente d'ailleurs non en militaire, mais en civil, la veste jetée nonchalamment sur l'épaule, en référence à sa promenade « nostalgique » dans la ville en 1974. Le débat qui a accompagné l'installation de la statue, puis son déplacement pour des raisons de politique urbaine quelques années plus tard, témoigne du fait, comme l'écrit V. Davis, que « le mythe et la mémoire peuvent être d'essence collective, leur signification demeure personnelle et sujette à controverses » (p. 129). Trente ans plus tard, les deux visites de Vladimir Putin, en tant que premier ministre, puis président, en 2010 et en 2011, confirment et renforcent la légitimité du mythe de Novorossijsk ; même si le président ne mentionne pas Brežnev lors de ses passages, se concentrant sur l'héroïsme des libérateurs de la ville de 1943, le résultat est qu'il existe désormais deux mythes complémentaires, un au niveau national, celui de la « Victoire », et un au niveau local, lié à l'ancien secrétaire général.

Dans la troisième partie, Vicky Davis se penche sur la dimension ritualiste et monumentale du mythe. Novorossijsk est, de ce point de vue, une « ville mémoire » par excellence, avec plus de deux cents monuments érigés en souvenir du conflit depuis 1960, qui avait vu l'inauguration du « square des héros », « épicentre mémoriel » de la ville, avec le mémorial de Malaja Zemlja (qui ne se trouve pas, contrairement à une idée reçue, sur le lieu même des combats), inauguré en 1982, peu de temps avant la mort de Brežnev. Ici aussi, l'auteur montre bien comment la population, « aiguillonnée » par une incitation d'en haut (en l'occurrence, le Komsomol et son périodique Komsomol'skaja Pravda), en est venue à créer un certain nombre de manières « alternatives », à la fois indépendantes et complémentaires, d'honorer les morts, comme le rituel de la beskozyrka (coiffe de marin soviétique), en hommage aux soldats d'infanterie marine morts sans sépulture.

Enfin, dans la quatrième partie (« rencontres intergénérationnelles »), V. Davis dissèque les stratégies destinées à " apprendre aux jeunes à se souvenir » et se penche notamment sur la notion de vospitanie, l'éducation au «patriotisme » qui a connu un développement sans précédent depuis les années 2008-2010, dont l'efficacité s'explique par le rapprochement des familles et de l'école, qui a intégré dans ses programmes l'apprentissage du patriotisme. V. Davis montre comment le « devoir de mémoire » est mis en place par les autorités locales et régionales supervisées par l'État fédéral. Pour l'auteur, le succès du vospitanie, une éducation autant historique que morale, s'explique par une politique intelligente qui incite les jeunes à participer activement à la recréation du passé, à une politique qui encourage la conservation et la transmission privée de la mémoire de la guerre pour son mythe global de « nation victorieuse », un réseau de « conseils d'anciens combattants » qui participent activement aux commémorations. En effet, contrairement à une tendance observée ailleurs en Russie et en Occident, la jeunesse de Novorossijsk 
se montre globalement très réceptive au mythe de la guerre et ne manifeste pas de signes de désaffection et d'apolitisme.

L'ouvrage de Vicky Davis est publié dans une collection qui a pour vocation, si l'on en croit l'éditeur, à « éclairer sous un jour nouveau l'histoire de la Russie », et ce, pour « tordre le cou aux confusions et aux malentendus qui abondent actuellement $»$ : une mission pédagogique donc, destinée au grand public ${ }^{5}$. Le livre est écrit dans une langue accessible, et répond donc à cette exigence. L'auteur convoque un grand nombre de références qu'elle intègre intelligemment au récit, montrant le haut degré de problématisation de l'ensemble. Çà et là cependant, on peut regretter des carences dans le choix du plan. Ainsi, V. Davis aurait été mieux inspirée de raconter en introduction l'histoire de Novorossijsk et de sa région, au moins au $\mathrm{XX}^{\mathrm{e}}$ siècle (la mention d'une république éphémère de Novorossijsk en 1905 est faite en passant à la fin du livre), et d'autre part, de donner plus de détails sur la campagne militaire et son contexte, pour comprendre pourquoi le culte de Malaja Zemlja sous Brežnev, érigé au niveau d'un tournant majeur de la guerre, fut tant discrédité par la suite.

D'autres points auraient pu aussi être plus systématiquement exposés, ainsi les autres « mythes de guerre » soviétiques, s'ils sont mentionnés ici ou là, auraient pu faire l'objet d'un comparatisme plus approfondi ; de même pour les comparaisons avec les autres pays, qui auraient pu faire l'objet d'une section à part. V. Davis aurait ainsi pu proposer une comparaison entre le mythe du débarquement à Novorossijsk et celui du débarquement de Normandie de 1944. Autre regret : les « carnets de Brežnev », publiés en 2016, n’ont pas été utilisés ici, or on y trouve plusieurs références à l'implication de Brežnev dans le façonnement du mythe de Novorossijsk. Les sources utilisées par V. Davis sur Brežnev lui-même sont un peu trop légères, puisque, outre des témoignages oraux, elle exploite des biographies russes qui, on le sait, ne sont absolument pas scientifiques, étant dépourvues de tout corpus de notes.

Plus gênant selon nous est le parti pris de départ de l'auteur, qui consiste à considérer que la dimension mensongère du mythe de la guerre n'est pas importante : ce qui lui importe est d'étudier son impact au sein de la population de Novorossijsk (p. 24). Or la dimension de falsification et de propagande mensongère des mythes est liée aux conditions de leur réception et on ne peut l'éluder. On ne peut comprendre la politique des autorités, locales, régionales et nationales, sans voir la nature et la raison du succès de ces falsifications. Enfin, la démonstration de V. Davis selon laquelle le mythe de guerre fait l'objet, chez la population de Novorossijsk, d'une quasi totale adhésion, est de nature à décevoir — on aurait été bien surpris du contraire pour cette ville, qui plus est en se fondant sur des entretiens accordés par des locaux à une Britannique, et ce, en pleine « nouvelle guerre froide »!

Pour aborder la question de la réception et le succès du mythe, l'auteur aurait peut-être pu commencer son récit avec l'incident de 2015 (qu'elle évoque brièvement dans la dernière partie), lorsque des jeunes filles de Novorossijsk avaient mis en ligne une vidéo où elles dansaient le « twerk », danse sensuelle dérivant du twist et du jerk, devant le mémorial de Malaja Zemlja, suscitant une émotion considérable. Deux d'entre elles ont écopé à cette occasion de la prison ferme, mais ont échappé à l'inculpation de « profanation $»^{6}$. Une « amorce » qui aurait pu frapper les imaginations, 
car non sans rappeler l'affaire des Pussy Riot - la « profanation » de l'église du Christ Sauveur de Moscou en 2013. Une enquête plus approfondie sur cette affaire aurait éventuellement pu déboucher sur des conclusions plus nuancées (pour ne pas dire moins naïves) que celles de l'auteur, sur « l'exceptionnalité » de Novorossijsk sur l'apparent consensus sociétal autour du mythe de la guerre. Mais à l'impossible nul n'est tenu, et en l'état, l'enquête de Vicky Davis représente déjà une contribution considérable à notre connaissance du « culte de la guerre » dans la Russie poutinienne.

1 - Sur Brežnev, l'ouvrage qui a relancé la recherche fut celui d'Edwin Bacon et Mark Sandle, dir., Brezhnev reconsidered, Palgrave, 2002. Pour les travaux plus récents, voir Susanne Schattenberg, Leonid Breschnew : Staatsmann und Schauspieler im Schatten Stalins. Eine Biographie, Cologne, 2017 (traduit en 2018 en russe aux éditions Rosspen) ; sur le contexte brejnévien, voir Dina Fainberg et Artemy Kalinovsky, dir., Reconsidering Stagnation in the Brezhnev Era : Ideology and Exchange, Lexington books, 2016. Sur la mémoire de la guerre, voir Nina Tumarkin, The Living and the Dead: The Rise and Fall of the Cult of World War II in Russia, Basic Books, 1994; Amir Weiner, Making Sense of War :The Second World War and the Fate of the Bolshevik Revolution, Princeton University press, 2001; Roger Markwick, Rewriting History in Soviet Russia : The Politics of Revisionist Historiography 1956-1974, Palgrave, 2001 ; Lisa Kirschenbaum, The Legacy of the Siege of Leningrad, 1941-1945 : Myth, Memories and Monuments, Cambridge University Press, 2006 ; Polly Jones, Myth, Memory, Trauma : Rethinking the Stalinist Past in the Soviet union, 1953-1970, Yale University Press, 2013 ; Shaun Walker, The Long Hangover : Putin's New Russia and the Ghosts of the Past, Oxford University Press, 2018. Voir aussi deux articles d'Olga Kucherenko, "That'll Teach'em to Love Their Motherland! : Russian Youth Revisit the Battles of World War II », The Journal of Power institutions in post-Soviet societies, 12/2011, URL: https://journals.openedition.org/pipss/3866 ; et «"Their Overdue Landing" : A View from the Eastern Front ", in Michael Dolski, Sam Edwards, John Buckley, dir., D-Day in History and Memory : The Normandy Landings in International Remembrance and Commemoration, University of North Texas Press, 2014, p. 221-255.

2 - Voir Anne Le Huérou et Elisabeth Sieca-Kozlowski, dir., Culture militaire et patriotisme dans la Russie d'aujourd'hui, Karthala, 2008; Serguei Oushakine, The Patriotism of Despair : Nation, War and Loss in Russia, Cornell University Press, 2009. Stephen M. Norris, Blockbuster History in the New Russia: Movies, Memory, and New Patriotism, Indiana University Press, 2012 ; Julie Hemment, Youth Politics in Putin's Russia : Producing Patriots and Entrepreneurs, Indiana University Press, 2015.

26 - Merle Fainsod, Smolensk under the Soviet Rule, Harvard University Press, 1958, traduit sous le titre Smolensk à l'heure de Staline, Fayard, 1967. Les mêmes archives ont été exploitées avec une conceptualisation plus moderne par Laurie R. Cohen, dans Smolensk Under the Nazis: Everyday Life in Occupied Russia, University of Rochester press, 2013. Un autre exemple célèbre de « monographie d'histoire locale » est l'ouvrage de Stephen Kotkin sur la ville de Magnitogorsk : Magnetic Mountain : Stalinism as a Civilization, University of California Press, 1997. Voir aussi l'ouvrage de Lisa Kirschenbaum cité précédemment.

3 - Un ouvrage qui aurait pu figurer dans la bibliographie, déjà très riche, de l'auteur, est : Graeme Gill, Symbols and legitimacy in Soviet politics, Cambridge University Press, 2011. 4 - Voir la présentation de la collection « Library of Modern Russia » sur https://www. ibtauris.com/Series/Library-of-Modern-Russia. La question de l'origine de ces « confusions et malentendus » (russe ou occidentale) est sciemment passé sous silence par I.B. Tauris l'objectif étant de séduire autant les prétendus « russophiles » que les « russophobes ».

5 - Voir par exemple le reportage de Radio Liberté sur https://www.youtube.com/ watch?v=spSwGhp3wyc (consulté le 17 novembre 2018). 
Andreï Kozovoï

Université de Lille 